\title{
RESOLUTION
}

The following resolution was proposed and accepted by the participants in IAU Sympósium No. 111:

"IAU Symposium No. 111 participants (The Calibration of Fundamental Stellar Quantities, Como, Italy, May 24-29, 1984) call the attention of the directors of observing facilities and members of the program selection committees to the basic importance of observing standard stars together with program objects, and urge them to allocate sufficient time for this purpose.

It is also necessary to allocate time for the establishment of new standards in some cases: for example, faint standards for large telescopes, or ultraviolet standards for space-borne telescopes. Failure to establish and re-observe frequently standard objects u1timately will compromise the quality of the work done at any observatory."

\section{DISCUSSION OF SUGGESTION BY CODE}

During the symposium, Dr. A. D. Code suggested that the assembled participants discuss the question: "For the non-specialist user, how does he know which catalogue to use, and how to use it? Should we get specialists to write critiques of catalogues, which perhaps, could be included with the material sent out by the Centre de Donées Stellaires?" This question was suggested as the basis of a possible resolution. It was the sense of the meeting that this was a very important question, and that any critiques of catalogues would have to be very carefully done. The discussion was concluded with acceptance of the suggestion by Hauck that the question be taken up by the Working Group for Astronomical Data, and be discussed by the General Assembly. 